\title{
CME-HSS Interaction and Characteristics Tracked from Sun to Earth
}

\author{
Stephan G. Heinemann ${ }^{1}(D)$ Manuela Temmer $^{1}$ (D) Charles J. Farrugia ${ }^{2}$. \\ Karin Dissauer $^{1}(\mathrm{D})$. Christina Kay ${ }^{3,4}(\mathrm{D})$. Thomas Wiegelmann ${ }^{5}$. \\ Mateja Dumbović ${ }^{1}$ (D) Astrid M. Veronig ${ }^{1,6}$ (D) Tatiana Podladchikova ${ }^{7}$. \\ Stefan J. Hofmeister ${ }^{1}$ (D) - Noé Lugaz ${ }^{2}$ (D) - Fernando Carcaboso ${ }^{8}$ (D)
}

Received: 16 April 2019 / Accepted: 27 August 2019 / Published online: 13 September 2019

(C) The Author(s) 2019

\begin{abstract}
In a thorough study, we investigate the origin of a remarkable plasma and magnetic field configuration observed in situ on June 22, 2011, near L1, which appears to be a magnetic ejecta (ME) and a shock signature engulfed by a solar wind high-speed stream (HSS). We identify the signatures as an Earth-directed coronal mass ejection (CME), associated with a C7.7 flare on June 21, 2011, and its interaction with a HSS, which emanates from a coronal hole $(\mathrm{CH})$ close to the launch site of the CME. The results indicate that the major interaction between the CME and the HSS starts at a height of $1.3 \mathrm{R}_{\odot}$ up to $3 \mathrm{R}_{\odot}$. Over that distance range, the $\mathrm{CME}$ undergoes a strong north-eastward deflection of at least $30^{\circ}$ due to the open magnetic field configuration of the $\mathrm{CH}$. We perform a comprehensive analysis for the CME-HSS event using multi-viewpoint data (from the Solar TErrestrial RElations $\mathrm{Ob}$ servatories, the Solar and Heliospheric Observatory and the Solar Dynamics Observatory), and combined modeling efforts (nonlinear force-free field modeling, Graduated Cylindrical Shell CME modeling, and the Forecasting a CME's Altered Trajectory - ForeCAT model). We aim at better understanding its early evolution and interaction process as well as its interplanetary propagation and related in situ signatures, and finally the resulting impact on the Earth's magnetosphere.
\end{abstract}

S.G. Heinemann

stephan.heinemann@ hmail.at

1 Institute of Physics, University of Graz, Universitätsplatz 5, 8010 Graz, Austria

2 Institute for the Study of Earth, Oceans, and Space, University of New Hampshire, Morse Hall, 8 College Road, Durham, NH 03824-3525, USA

3 Solar Physics Laboratory, NASA Goddard Space Flight Center, Greenbelt, MD, USA

4 Dept. of Physics, The Catholic University of America, Washington DC, USA

5 Max-Planck-Institut für Sonnensystemforschung, Justus-von-Liebig-Weg 3, 37077 Göttingen, Germany

6 Kanzelhöhe Observatory for Solar and Environmental Research, University of Graz, 9521 Treffen, Austria

7 Skolkovo Institute of Science and Technology Skolkovo Innovation Center, Building 3, Moscow 143026, Russia

8 Dpto. de Física y Matemáticas, Universidad de Alcalá, 28805 Alcalá de Henares, Madrid, Spain 
Keywords Corona - Coronal holes · Coronal mass ejections, initiation and propagation · Coronal mass ejections, interplanetary - Coronal mass ejections, low coronal signatures · Magnetic fields, photosphere $\cdot$ Magnetosphere, geomagnetic disturbances

\section{Introduction}

Coronal mass ejections (CME) frequently pass over Earth at an average rate of $1-2$ events per month but with significant variations throughout the solar cycle (Richardson and Cane, 2010). They are the major cause of strong geomagnetic effects, especially during solar maximum (e.g., Farrugia, Burlaga, and Lepping, 1997; Liu et al., 2014). Besides CMEs, stream interaction regions (SIR) and, if persistent for several solar rotations so-called co-rotating interaction regions (CIR), structure interplanetary space. It is the interaction between solar wind high speed streams (HSS), emanating from coronal holes $(\mathrm{CH})$, and the slow solar wind ahead, which forms compression regions, shocks and rarefaction regions, causing recurrent geomagnetic effects on Earth (see, e.g., Alves, Echer, and Gonzalez 2006, Verbanac et al. 2011, Vršnak et al. 2017, Yermolaev et al. 2018, Richardson 2018). While HSSs pose a continuous outflow, CMEs abruptly disrupt the rather steady solar wind structure, causing deviations (preconditioning) from the quiet solar wind conditions over the duration of several days (Temmer et al., 2017).

The interaction of CMEs with the solar wind, especially HSSs, may significantly change the CME properties en route through the heliosphere. The embedded flux rope may deform, kink or rotate (Manchester et al., 2004; Riley and Crooker, 2004; Wang et al., 2006; Yurchyshyn, 2008; Isavnin, Vourlidas, and Kilpua, 2013), erode due to reconnection (Dasso et al., 2006; Ruffenach et al., 2012, 2015; Lavraud et al., 2014; Wang et al., 2018), be deflected (Wang et al., 2004, 2014a, 2016; Manchester et al., 2005; Kay, Opher, and Evans, 2013, 2015; Zhuang et al., 2019) and may be related to increased turbulence in the sheath region (Lugaz et al., 2015; Kilpua, Koskinen, and Pulkkinen, 2017). Fast solar wind may also cause the CME to speed up, hence, shortening the propagation time between Sun and Earth. Nieves-Chinchilla et al. (2012) investigated in detail the rotation of a CME as it propagates through the heliosphere. It is understood that changes in CME properties differ strongly for processes taking place already low in the corona compared to those happening in interplanetary space (Wang et al., 2014b; Winslow et al., 2016). All these effects alter the initial CME properties observed close to the Sun making predictions of arrival time and geoeffectiveness a complex endeavor (Richardson, 2018). This also shows that the ambient solar wind plays an important role for CME propagation, and that it is necessary to study and understand the interaction processes already from its source on the Sun and related 1 AU signatures.

Previous studies of CME-HSS interaction events often focused on HSSs catching up with a CME deforming and compressing it (Winslow et al., 2016; He et al., 2018). In this study the CME appears to be propagating behind the SIR within the HSS, giving rise to a number of peculiarities in the observed in situ signatures. By studying in detail this CMEHSS interaction event we aim to unravel the complex physical processes related to a $\mathrm{CME}$ propagating in a HSS starting from the CME eruption site close to the HSS related $\mathrm{CH}$. To do so, we investigate the Sun-Earth chain of a distinct and well-observed CME-HSS interaction event combining remote sensing observations, in situ measurements at $1 \mathrm{AU}$ and modeling efforts. We further investigate the effects on the Earth's magnetosphere as a consequence of the CME-HSS interaction. 


\section{Motivation}

Starting on June 22, 2011, we observed an intriguing configuration of plasma and magnetic field in situ signatures near L1. We find a SIR signature with a clear stream interface (SI) followed by a shock within the HSS on June 23, 2011 (Figure 1). After a short standoff distance, the magnetic ejecta (ME) can be identified propagating with the same speed as the ambient solar wind of the HSS. This gives rise to the two major questions which we address in this study:

- How can these unique shock characteristics be explained? Usually, a shock within a high speed environment, like a HSS, would either quickly propagate through or would dissipate in the case it is not driven. The intuitive solution would be that the shock is driven by the ejecta.

- Is the shock driven? Judging from the short standoff distance between the shock and the ejecta and that the shock signature is found in the middle of the HSS, one would suspect so. However, the speed of the ejecta is equal to the speed of the HSS, but the speed of the shock is higher, which indicates that it is not driven at that distance.

To answer these questions we track the CME from 1 AU back through the interplanetary space to its origin on the solar disk and examine what processes may produce such in situ signatures and what geomagnetic effects they cause. The paper is structured as follows: In Section 3 the in situ signatures are investigated. In Section 4 the CME launch and propagation is analyzed using remote sensing data and various modeling efforts. Section 5 presents the resulting geomagnetic effects. In Section 6 the results are discussed and summarized in Section 7.

\section{In Situ Signatures}

Using OMNI ${ }^{1}$ data from the Advanced Composition Explorer (ACE; Stone et al. 1998) and the Global Geospace Science Wind satellite (Acuña et al., 1995), we investigate the properties of the solar wind and CME plasma at a distance of about $1 \mathrm{AU}$.

Figure 1 shows a 24-hr period from 18 UT, June 22 to 18 UT, June 23, 2011. From top to bottom, we show the proton number density and the alpha-to-proton number density ratio, the bulk speed, the temperature and expected proton temperature for normal solar wind expansion (Lopez, 1987), the total perpendicular pressure (Russell, Shinde, and Jian, 2005) and dynamic pressure including the $\alpha$ particles, the components of the magnetic field in GSM coordinates, the total magnetic field, and the proton $\beta$ and the Alfvèn Mach number. From the proton bulk speed one can identify a slow stream followed by a fast one. The stream interface occurs at around 20 UT, June 22 shown by the orange vertical guideline. The SI was identified based on the sharp drop of the density compression and the sharp rise in the temperature as shown in Figure 1. Additionally we observe a gradient in the flow, where the east-west flow changes sign (not shown). The following high speed solar wind stream is interrupted at 03:06 UT by simultaneous sharp rises in the density, temperature, speed and magnetic field, corresponding to a shock structure. Its strength is moderate, with a density ratio of $\sim 2$ and in situ a field compression of $\sim 1.5$. By using the coplanarity theorem (Colburn and Sonett, 1966; Abraham-Shrauner, 1972; Abraham-Shrauner and Yun, 1976),

\footnotetext{
${ }^{1}$ https://omniweb.gsfc.nasa.gov/.
} 


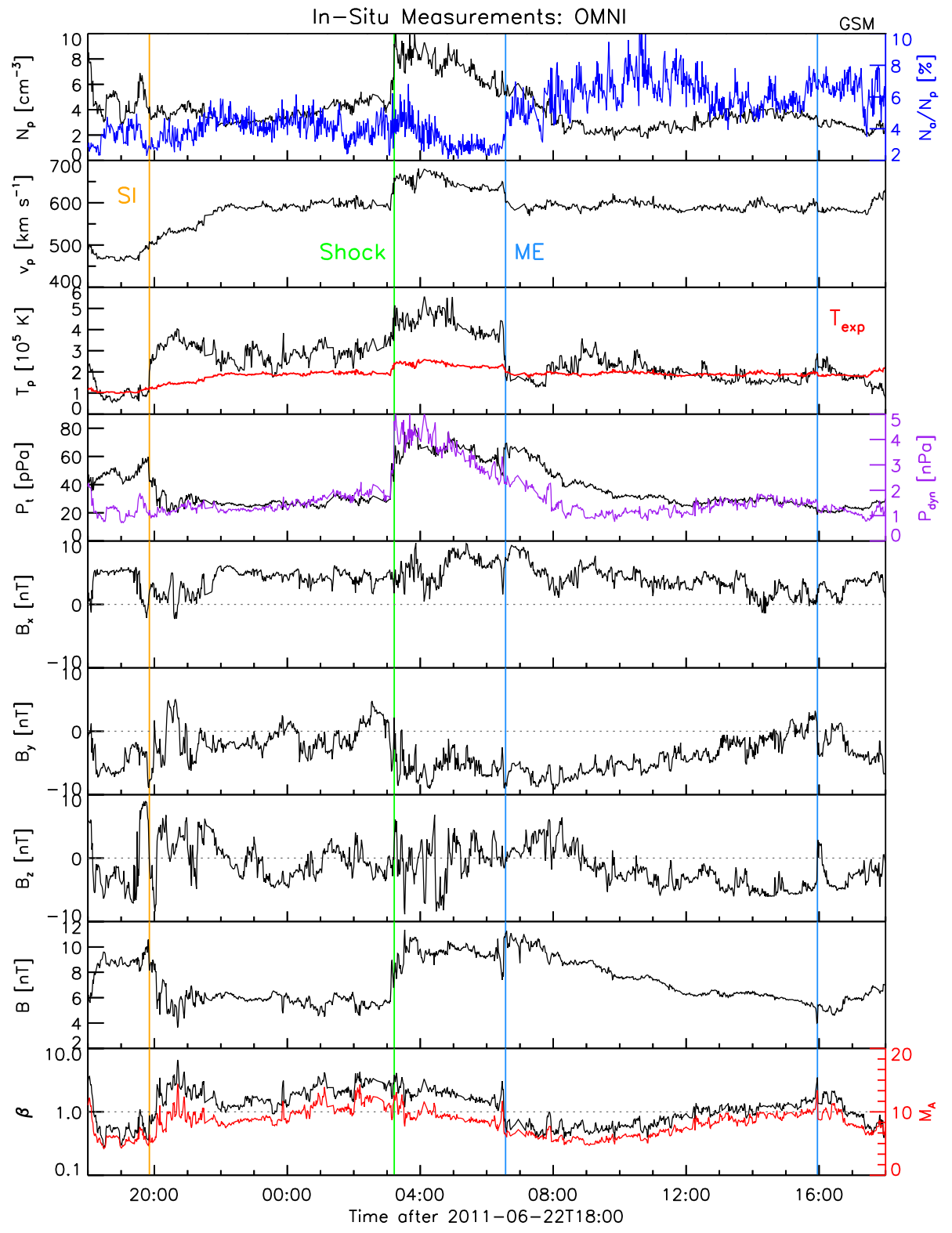

Figure 1 In situ measured Wind and ACE data from the OMNI database of a $24 \mathrm{~h}$ time interval during the HSS and CME arrival starting at June 22, 2011, 18 UT. From top to bottom: Proton density (black) with the $\alpha$-particle ratio overlayed (blue); proton velocity; proton temperature (black) with the expected temperature in red; total perpendicular pressure (black) and the dynamic pressure (purple); magnetic field components (panel 5-8); plasma- $\beta$ (black) and the Alfvénic Mach number (red). The vertical guidelines represent the start times of the stream interface (orange), the shock (green) as well as the interval identified as the magnetic ejecta (blue lines) as derived from the data in this figure. 


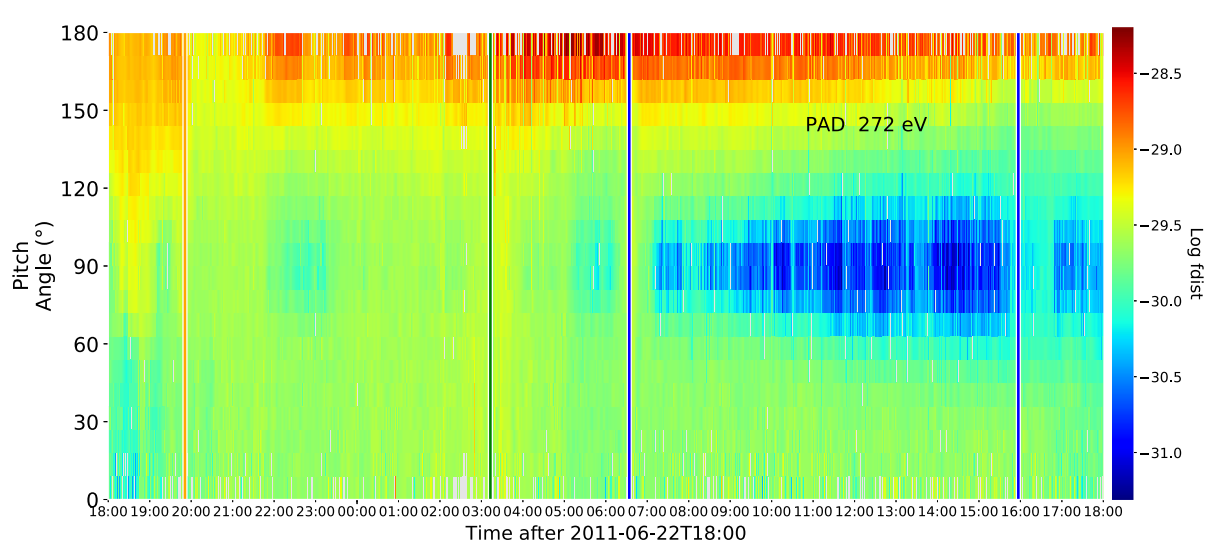

Figure 2 Suprathermal electrons pitch-angle distribution observed by ACE for the $272 \mathrm{eV}$ energy channel, time-shifted to match the OMNI data. A bidirectional distribution can be observed during the transit of the magnetic ejecta. The vertical guidelines are the same as shown in Figure 1.

which assumes that the magnetic field on both sides of the discontinuity and the shock normal all lie in the same plane, we calculate the shock normal. The shock normal speed we derive using the mass conservation equation across the discontinuity (e.g., see Paschmann and Daly, 1998, and the references therein). Using the magnetic coplanarity (Colburn and Sonett, 1966) we calculate the shock normal velocity to be $\sim 710 \mathrm{~km} \mathrm{~s}^{-1}$. The angle between the upstream magnetic field and the shock normal $\left(\theta_{B u, n}\right)$ is $\sim 30^{\circ}$ which makes it a quasi-parallel shock. When using the mixed mode coplanarity (Abraham-Shrauner, 1972) to calculate the shock normal, we derive $v_{\text {shock }} \sim 678 \mathrm{~km} \mathrm{~s}^{-1}$ with $\theta_{B u, n} \sim 34^{\circ}$. Behind the shock the elevated high speed continues for $\sim 3.75 \mathrm{hrs}$. Then a structure with high magnetic field strength, low proton temperature, low beta and Alfvén Mach number, and increased alpha-to-proton number density ratio is present.

Figure 2 presents the pitch angle distribution of suprathermal electrons in the ACE $272 \mathrm{eV}$ channel showing isotropic flux during the shock arrival and the transition from the shock-sheath to the magnetic ejecta, which is expected. In this interval, we identify as the magnetic ejecta the period during which the electrons show bidirectionality, suggesting a closed magnetic structure (e.g., Montgomery et al. 1974, Gosling et al. 1987, Carcaboso Morales et al. 2018). This strongly supports the interpretation of a flux rope within the HSS.

We note that the bulk speed before the shock and after the shock-sheath is the same, which suggests that the magnetic ejecta is currently not driving the shock. This can be interpreted as follows: the $\mathrm{CME}$ is embedded and dragged along with the co-rotating interaction region (CIR), which is an intriguing feature.

\section{Remote Observations \& Modeling}

Using white-light as well as extreme ultra-violet (EUV) observations obtained by the Sun Earth Connection Coronal and Heliospheric Investigation (SECCHI; Howard et al. 2008) suite on-board the Solar TErrestrial RElations Observatories (STEREO-A/B; Kaiser et al. 2008) we are able to track the CME back to its origin on the solar disk. Figure 3 shows the propagation of the CME along the equatorial cut as seen by STEREO-A's Heliospheric Imager 1 and 2 (HI1, HI2) as well as its coronagraph (COR2) obtained in the form of a 
Figure $3 \mathrm{~J}$-map of the ICME as seen in COR2, HI1 and HI2. The ICME kinematic is marked by the red dotted line and in situ arrival of the shock by the red dashed line. This feature is manually tracked back to the corresponding CME launch time at the Sun around 02 UT on June 21, 2011.

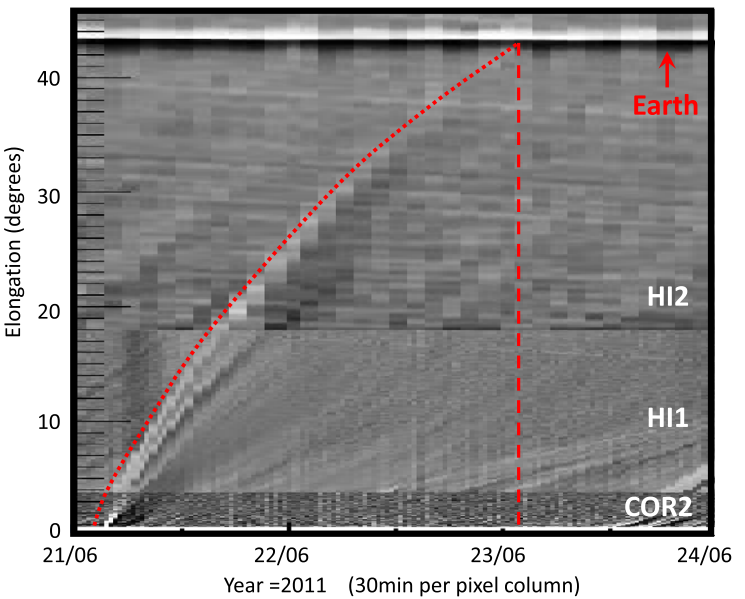

J-map. We estimate the launch time at the Sun to be around June 21, 2011, 02 UT. This date coincides very well with an observed gradual C7.7 flare from the active region (AR) 11236 (location: N17/W12) starting at 01:22 UT on June 21, 2011. The flare can be associated with an Earth-directed halo CME whose signatures are subsequently observed by the Solar Dynamics Observatory (SDO; Pesnell, Thompson, and Chamberlin 2012), the Solar and Heliospheric Observatory (SOHO; Domingo, Fleck, and Poland 1995) and both STEREOs. A medium-size coronal hole $(\mathrm{CH})$ is located in the south-west of the AR that we associate with the CIR observed in situ near 1 AU on June 22, 2011. Figure 4 shows the GOES soft X-ray flux around the time of the flare (top), which features a very gradual increase over nearly $2 \mathrm{hrs}$ before the peak in the soft X-ray flux $(1-8 \AA)$ of $7.7 \times 10^{-7} \mathrm{~W} / \mathrm{m}^{2}$ is reached at around 03:25 UT. The panels (from left to right) show the solar corona in the $211 \AA$ EUV filter taken by the Atmospheric Imaging Assembly (AIA; Lemen et al. 2012) on-board SDO, before, during and after the flare. Before the flare starts, the AR and the $\mathrm{CH}$ to the south can be well observed. As the flare progresses, the post-eruptive loop system and coronal dimming regions evolve related to the magnetic field restructuring and density depletion due to the erupting flux rope structure (see e.g., Dissauer et al., 2019).

\subsection{NLFFF Model}

To qualitatively describe the configuration of the corona that leads to the non-radial evolution of the CME, we study the ambient magnetic field configuration of the Sun. We use synoptic vector magnetograms from the Heliospheric and Magnetic Imager (HMI, Schou et al. 2012, Couvidat et al. 2016) on-board SDO as input for a global nonlinear force-free field (NLFFF) model. The method was originally proposed in Cartesian geometry by Wheatland, Sturrock, and Roumeliotis (2000), but here we use the spherical optimization code developed by Wiegelmann (2007) and adjusted for the use of synoptic vector maps in Tadesse et al. (2014). As boundary condition we use a synoptic vector map for Carrington rotation 2111, which has been observed between June 05, 2011, and July 03, 2011.

The left panel of Figure 5 shows the resulting two-dimensional open field map at $1 \mathrm{R}_{\odot}$ $(f(\theta, \phi))$ where yellow underlying contours corresponds to positive and blue underlying contours to negative polarity footpoints of open field lines. Overlayed is the NLFFF-model (Br) at a surface height of $r=2.5 \mathrm{R}_{\odot}$ with red representing positive polarity and green 

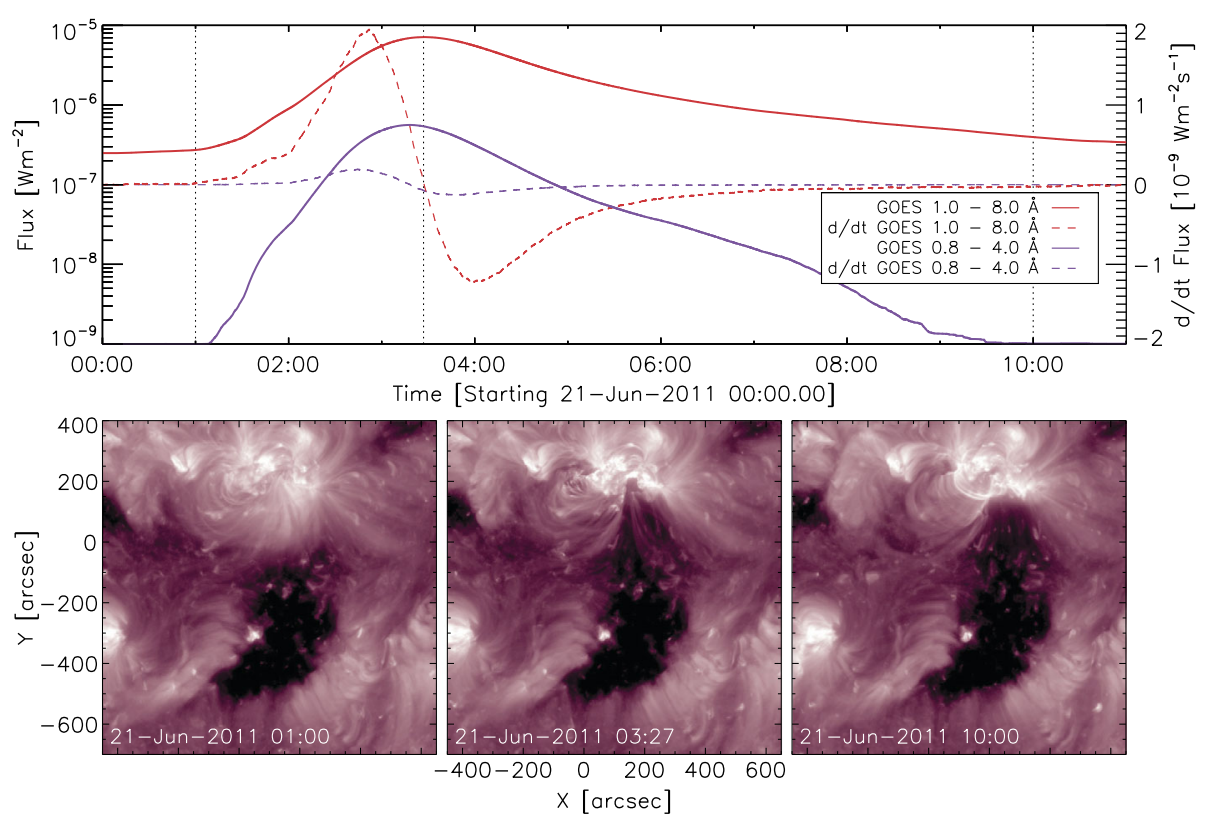

Figure 4 Top: GOES soft X-ray fluxes and time derivative of the observed C7.7 flare. Bottom: AIA/SDO $211 \AA$ images with the field of view centered around the source AR (N17/W12) and the CH located to the south. The image recording times are marked as the dotted vertical lines in the GOES soft X-ray profile and represent the configuration of the solar corona before, during and after the flare.

representing negative polarity. We see that the northern hemisphere has a negative polarity and, vice versa, the southern hemisphere has a positive polarity. Near the flux center of the flare we find open field footpoints to the south which are well observed in EUV as the $\mathrm{CH}$ (right panel of Figure 5). The open field footpoints to the west, indicative of the extension of the $\mathrm{CH}$, might be obscured as a consequence of nearby bright loops, and they are therefore not well visible as a dark region in EUV. The identified open field footpoints most likely form a magnetic potential barrier towards south and west, which the CME cannot easily cross due to the frozen-in condition in the coronal plasma. However, towards the north and east the CME can freely expand.

\subsection{Coronal Dimmings and CME Launch}

After establishing the conditions of the global solar magnetic configuration around the AR of interest, we analyze the evolution of coronal dimmings associated with the CME footpoints anchored at the surface. Coronal dimmings are regions of strongly reduced emission observed in EUV and SXRs (Hudson, Lemen, and Webb, 1996; Sterling and Hudson, 1997; Thompson et al., 1998, 2000). They are a signature of the density depletion that is caused by the plasma expansion and evacuation during the early CME eruption. Bipolar coronal dimmings are generally interpreted to represent the footprints of CMEs in the low corona (e.g. Thompson et al. 2000, Dissauer et al. 2019) and can be used as proxies for studying the initial CME behavior.

In order to properly track the dimming regions, we use a thresholding technique applied on logarithmic base-ratio EUV images. This also allows us to identify so-called secondary 


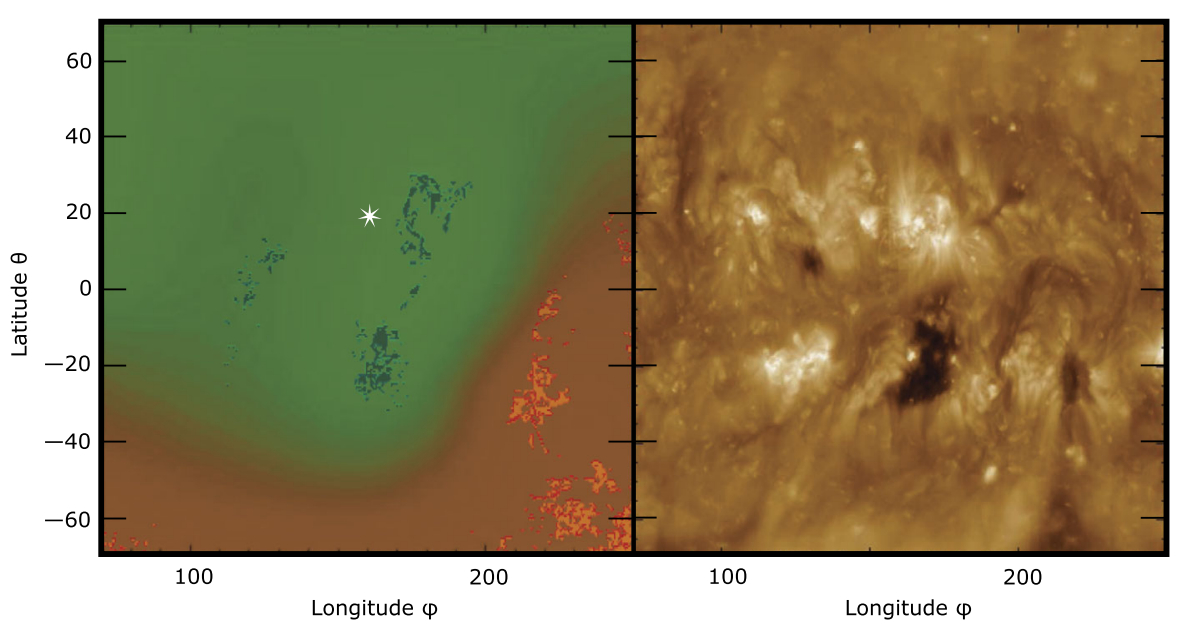

Figure 5 Left: CR2111, NLFFF-model at $r=1 \mathrm{R}_{\odot}$ : Two-dimensional open field map $f(\theta, \phi)$ where $f=1$ (yellow underlying contours) corresponds to positive and $f=-1$ (blue underlying contours) to negative polarity footpoints of open field lines. $f=0$ are areas hosting closed field lines. The NLFFF-model (Br) at $r=2.5 \mathrm{R}_{\odot}$ is overlayed, with the red shaded area representing positive polarity and green representing negative polarity. The white star marks the location of the AR. Right: Synoptic image (CR2111) of the solar corona observed in the SDO/AIA $193 \AA$ filter for the same field of view.

dimmings, which are mapping the overlying magnetic field that is expanding and erupting. They are of special interest, since they mostly reflect the propagation direction of the CME in its early evolution phase. To capture the full extent of coronal dimmings over time, we derive cumulative dimming masks. These masks contain all dimming pixels that are detected below a certain threshold and over a given time range. In this way different parts of the dimming that may grow and recover, e.g. due to the associated flare, are also included in the detection. For further details on the method we refer to Dissauer et al. $(2018,2019)$.

Figure 6 shows the evolution of the CME-associated dimming region, where each pixel is color-coded by the time of its first detection. Dark blue pixels are detected earlier than light blue pixels, respectively. The three contours represent the time evolution of the dimming region during its main impulsive phase, while the $C M E$ is propagating below $2 \mathrm{R}_{\odot}$, at $02: 10$ UT (red), 02:34 UT (green), and 02:58 UT (magenta), respectively. The dimming mainly grows/spreads towards the south-east and south until 03:00 UT (marked as black arrows) in the reference frame of the flux center of the flare (yellow asterisk). This clearly shows that the CME was launched from the southern part of the AR, evolving into the direction of the $\mathrm{CH}$. The eastward and south-westward spread may be caused by the CME lateral expansion.

Figure 7 gives the stereoscopic limb view of the CME using STEREO-A EUVI $195 \AA$ running-difference images. We observe a clear southward propagation direction of the CME during its launch, which is in agreement with the early evolution of the associated coronal dimming. The red arrows indicate the observed propagation direction of the CME apex in the lower corona, which was estimated visually. It follows the trend shown in the latitudinal propagation profile of Figure 8. Following the CME evolution further on, one can clearly see that the CME gets deflected towards north over the distance range $1.3-3 \mathrm{R}_{\odot}$, indicating an interaction with the open field from the $\mathrm{CH}$. 


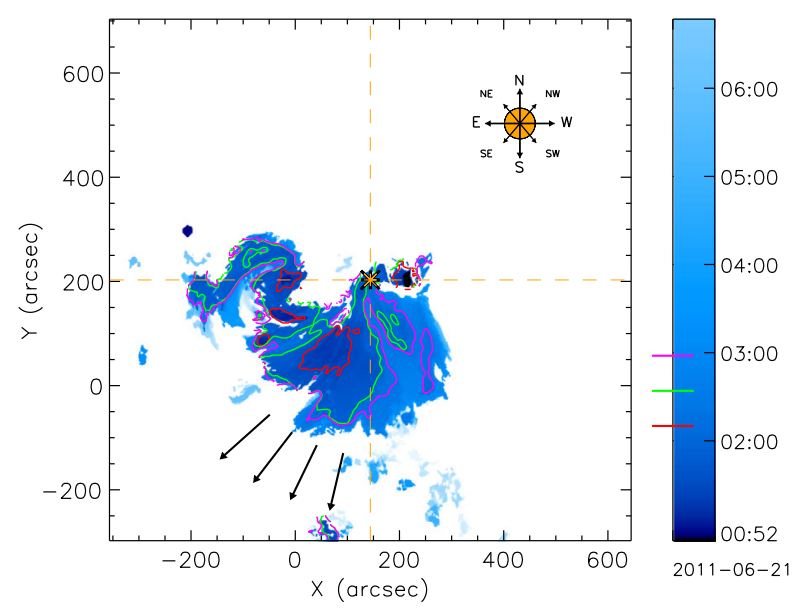

Figure 6 Evolution of the coronal dimming caused by the CME. Each pixel is color-coded by the time of its first detection, where darker pixel represent an earlier detection time than lighter ones. The contours represent the size of the dimming region at three timesteps (as indicated by the colored lines in the colorbar). The contours are at 02:10 UT, 02:34 UT and 02:58 UT, which represent the impulsive evolution phase of the CME (up to $1 \mathrm{R}_{\odot}$ above the solar surface). The arrows indicate the major evolution direction of the dimming. The image is centered at the flux center of the flare (yellow asterisks).

\subsection{ForeCAT and GCS}

To further study the propagation of the CME we use two modeling approaches, the Forecasting a Coronal mass ejection's Altered Trajectory (ForeCAT; Kay, Opher, and Evans 2013, 2015) and the Graduated Cylindrical Shell (GCS; Thernisien, Howard, and Vourlidas 2006, Thernisien, Vourlidas, and Howard 2009, Thernisien 2011) model. ForeCAT uses a Potential Field Source Surface model (PFSS; e.g. see Schatten, Ness, and Wilcox 1968) of the solar magnetic field to calculate the propagation, deflection and expansion of a CME on the basis of global magnetic pressure and tension. For further information on the model we refer to Kay, Opher, and Evans (2015).

Using the GCS model, we reconstruct the CME flux rope (FR) for 9 timesteps between 02:30 UT and 04:45 UT using COR1 and COR2 images from the SECCHI suite on both STEREO spacecraft in combination with LASCO C2 and C3 images (Brueckner et al., 1995). For the GCS reconstruction, which was fitted to best represent the white-light features of consecutive time steps, we derived large changes for latitude and longitude. This clearly shows the non-radial ejection and further deflection behavior of the CME due to its interaction with the open field of the HSS in the corona. Table 1 shows the fitted GCS parameters.

\subsubsection{Initial CME Propagation Results from ForeCat and GCS}

Figure 8 shows results of ForeCAT ensemble modeling (Kay and Gopalswamy, 2018) in comparison to the GCS FR parameters. The two top panels show the latitude of the CME axis and the two bottom panels show the longitude in Stonyhurst coordinates. We run an ensemble of 100 ForeCAT simulations with slight variations in the initial CME position and orientation. The blue line shows the seed value of the ensemble - the initial value that determines the center of the ensemble parameter range and our best guess at the true initial 


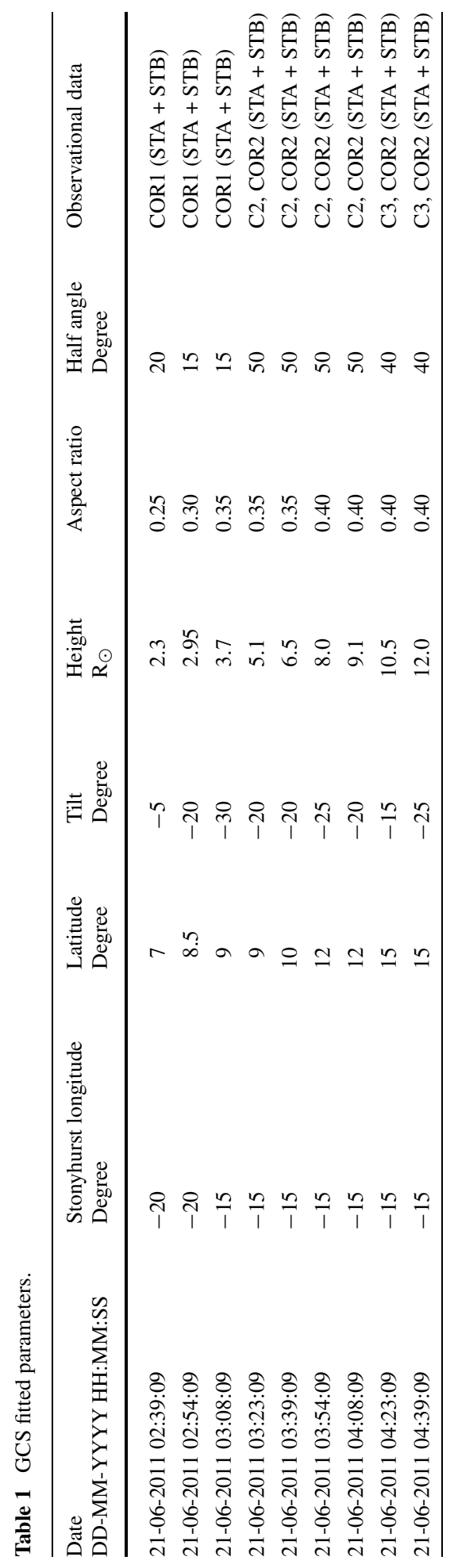



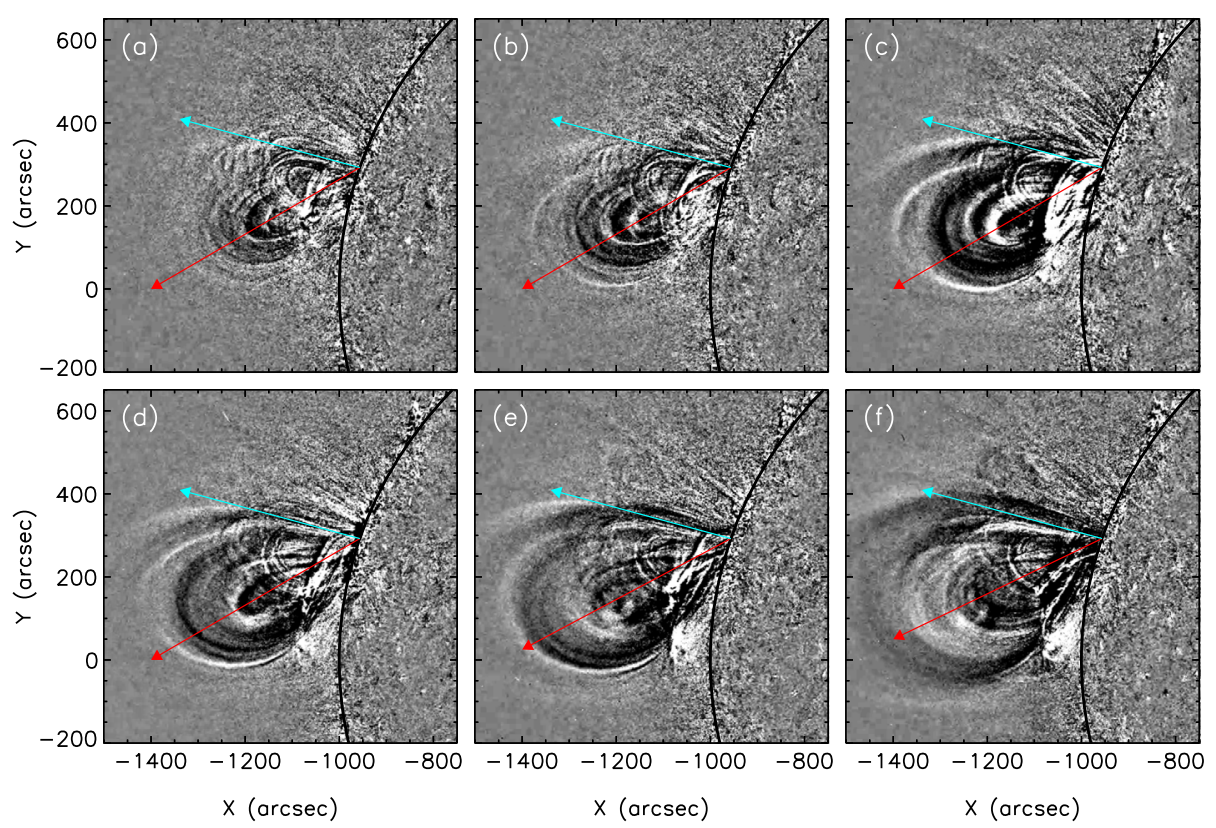

Figure 7 Series of running difference images of the CME early evolution observed by EUVI/STEREO-A in $195 \AA$. The images are from 01:58 UT (a), 02:03 UT (b), 02:10 UT (c), 02:15 UT (d), 02:20 UT (e) and 02:25 UT (f) and show a clear southward propagation of the CME (red arrows) in the first panels (a-d). In the last two panels a slowing down of the southward motion can be seen. The cyan arrows indicate the radial direction.

position and orientation. The dashed lines represent the median values, and the dark gray regions are one standard deviation about that. The red dots are the GCS values with error bars of $\pm 5^{\circ}$ for latitude and $\pm 10^{\circ}$ for longitude. The left panels show the results of the standard ForeCAT model, whereas in the right panels we included lateral overexpansion of the CME as well as artificial scaling of the magnetic field to simulate the compression of the field lines of the HSS. To simulate the compression we scaled the magnetic field strength in the direction of the $\mathrm{CH}$ by a scaling factor $S=1+\left(R-R_{0}\right) / 0.15 \mathrm{R}_{\odot}$ which was estimated empirically, where $R_{0}$ is the initial height of the CME nose.

We find the model CME to propagate from the northern hemisphere southwards and from west to east. After the initial phase the model suggests a near constant propagation direction at a latitude of $\sim 7^{\circ}$ north and a longitude of $\sim 10^{\circ}$ east. Starting at a height of about $2.3 \mathrm{R}_{\odot}$ GCS reconstructions are available. The GCS model parameters are very similar to the ForeCAT results. There is a slight eastward offset in the longitude but within the error bars. Only in the latitude we do derive a significant difference between the two results: the GCS reconstruction shows the values continuously rising (northward motion of the CME due to interaction with the HSS) which we cannot derive from the results of the standard ForeCAT model (Figure 8, left panel), which uses a static background magnetic field to model the deflection of a CME. The interaction of a CME and a HSS, however, involves dynamic processes in which, among others, the open magnetic field structure of the HSS will be compressed and the magnetic pressure increases to a point where the CME can no longer expand or propagate in that direction, which could lead to a change in the CME's trajectory. We see this northward motion is reproduced when we include the overexpansion and external compression in ForeCAT. 

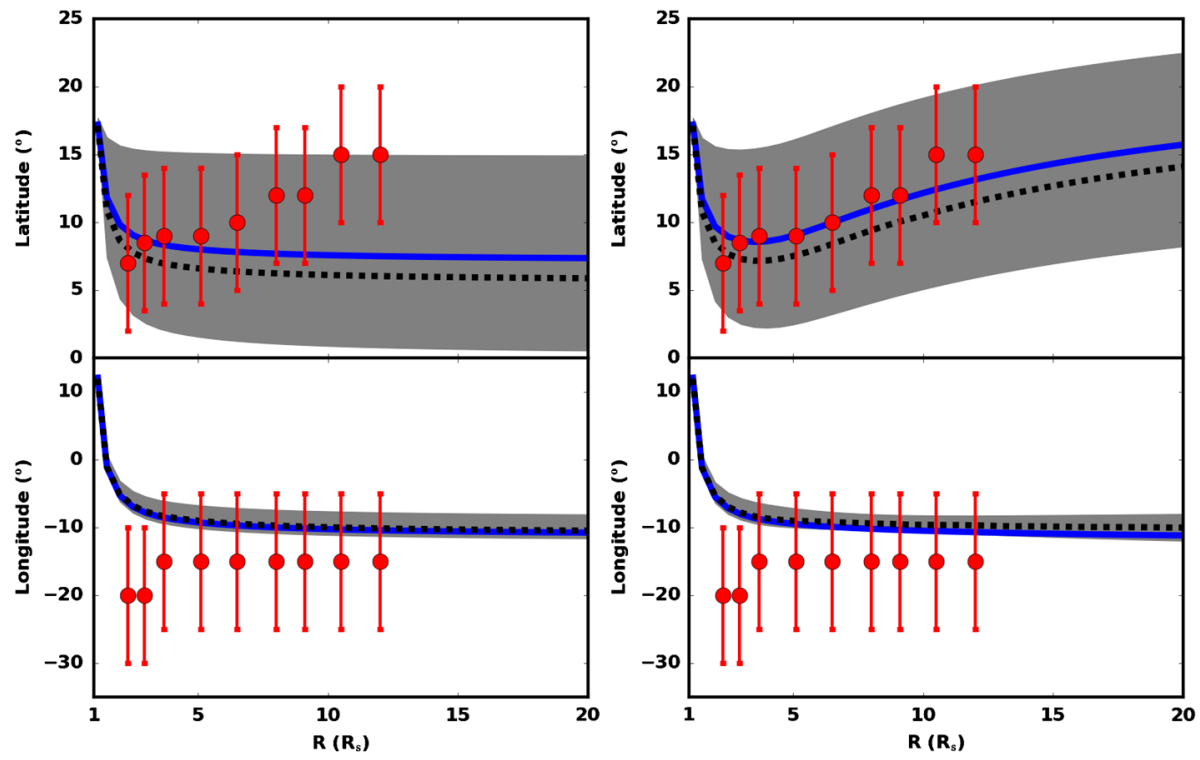

Figure 8 Results of ForeCAT ensemble modeling in comparison to the GCS flux rope parameters. The top panels show the latitude and the bottom ones the longitude (Stonyhurst) of the CME flux rope. The blue line shows the seed value of the ensemble. The dashed line represents the median values, and the dark gray regions are one standard deviation about that. The red dots are the GCS values. The left panels show the results of the standard ForeCAT model, whereas in the right panels we included lateral overexpansion of the CME as well as artificial scaling of the magnetic field to simulate the compression of the HSS.

\subsubsection{Flux Rope Evolution Using GCS Results}

Using the last GCS reconstruction at 04:45 UT on June 21, and the near 1 AU measured in situ signatures of the magnetic ejecta (duration, average speed) we estimate the expansion factor of the FR in interplanetary space (IP). Assuming self-similar expansion at an arbitrary rate constrained by the initial (GCS) and final size (in situ). We neglect the effects of the CME-HSS interaction in IP space on the CME trajectory and shape due to low plasma densities and magnetic fields. Based on the GCS fitting results, we estimate that the initial FR radius at $13 \mathrm{R}_{\odot}$ is $3.7 \mathrm{R}_{\odot}$. From the in situ measurement at 1 AU, we estimate the FR radius to be $31 \mathrm{R}_{\odot}$. The FR radius we estimate is the cross section along the Sun-Earth line, enabling us to investigate the geoeffective part of the magnetic structure. We note that this is the length along the observational path which means that it may not correctly represent the FR radius. The FR radius may be misrepresented in particular when the cross section is a skimming trajectory along the edge or a trajectory parallel to the FR axis. As we cannot clearly define the trajectory of the spacecraft through the magnetic structure (e.g. force-free flux tube fitting results are inconclusive due to the low magnetic field strength in the magnetic ejecta), we assume that the observational path is a reasonable estimate of the FR radius but we also have to consider that the uncertainties are large. Using a power-law equation for the increase of FR size with heliospheric distance given by Dumbović et al. (2018) adapted from a more general expression by Démoulin et al. (2008) and constrained by the derived GCS and in situ results we estimate the power-law index $n_{A}$, i.e. the expansion factor to $n_{A}=0.51_{+0.14}^{-0.13}$. We note that this resulting value lies on the lower end of the range obtained by statistical studies (e.g., see Gulisano et al., 2012; Wang et al., 2015) and indicates a rela- 
tive slow increase in FR size or that the in situ spacecraft passed through only a smaller part of the FR.

Additionally, assuming a constant axial magnetic flux we can estimate the drop-rate of the central magnetic field strength, assuming that it follows a power-law behavior with the power-law index $n_{B}$ (see Dumbović et al., 2018). We expect $n_{B}$ to be $1.02_{+0.28}^{-0.26}$, which again is on the lower end of the range observed in statistical studies (Gulisano et al., 2012) and indicates a quite slow drop of the magnetic field strength within the FR. With a magnetic field strength of $10 \mathrm{nT}$ at $1 \mathrm{AU}$ we estimate the initial magnetic field strength at $13 \mathrm{R}_{\odot}$ to be $0.002 \mathrm{G}$ and the toroidal magnetic flux at $1 \mathrm{AU}$ to be $\phi=1.63_{+0.85}^{-0.63} \times 10^{20} \mathrm{Mx}$. This value is at the lower end of the expected range of typical ICME fluxes of $10^{20}$ to $10^{22} \mathrm{Mx}$ for $\mathrm{C}$ to X-class events (e.g., DeVore, 2000; Qiu et al., 2007; Wang et al., 2015; Temmer et al., 2017). With such small expansion, we would expect the B field at 1 AU to be high (since the CME did not expand much) but in fact, $\mathrm{B}_{1 \mathrm{AU}} 10 \mathrm{nT}$, which is low. So either the initial field was very low to begin with and the in situ spacecraft only encountered part of the FR or $n_{A}$ does not reflect $n_{B}$.

\subsection{CME Kinematics in the Sun-Earth Line}

After analyzing the initial solar configuration, the launch and the start of the CME-HSS interaction using observations and models, as next step we investigate the propagation of the CME in the direction of Earth. We derived the height-time profile of the CME from multiple sources. We manually tracked the CME front in the equatorial plane using EUVI, COR1, and COR2 image data separately from STEREO-A and -B spacecraft. In addition, we obtained the height-time profile from the intersection of the front of the GCS reconstructed shell with the Sun-Earth line.

Figure 9 shows the derived CME kinematics (top to bottom: height-time, velocity and acceleration profile). The dots are the measurements (height-time) and their direct numerical time derivatives (velocity, acceleration). To obtain robust estimates of the corresponding velocity and acceleration profiles, we first smooth the height-time curves and then derive the first and second time derivatives. The smoothing algorithm is based on the method presented in Podladchikova, Van der Linden, and Veronig (2017), extended toward non-equidistant data. The algorithm optimizes between two criteria in order to find a balance between data fidelity, i.e., the closeness of the approximating curve to the data, and smoothness of the approximating curve. From the acceleration profiles obtained in this way, we then interpolate to equidistant data points based on minimization of the second derivatives, and reconstruct the corresponding velocities and height profiles by integration (solid lines). The estimation errors of kinematic profiles are obtained by representing the reconstructed CME height, velocity and acceleration as an explicit function of original CME height-time data in the assumption that STEREO-A and STEREO-B height errors are $1.5 \%$ of height, and $3 \%$ of height for GCS data. In the height-time diagram we derive that the CME is propagating from east (closer to STEREO-A) towards west (getting closer to STEREO-B) because of the initial offset between the black and blue line which cross later. The GCS model results are in good agreement with the direct measurements. The fit to the GCS height-time profile was done assuming that the acceleration has already subsided.

The velocity and acceleration profiles show the impulsive phase around 02:20 UT followed by a slower acceleration, which subsides over time. Beyond a height of about $3 \mathrm{R}_{\odot}$ the acceleration phase is finished and the CME propagates outwards at a speed of $800-$ $1000 \mathrm{~km} \mathrm{~s}^{-1}$ which then seems to adjust to the speed of the surrounding HSS. Near Earth, the in situ measured speed of the magnetic ejecta was $\sim 600 \mathrm{~km} \mathrm{~s}^{-1}$ which was roughly equal to the speed of the HSS (see Figure 1). 


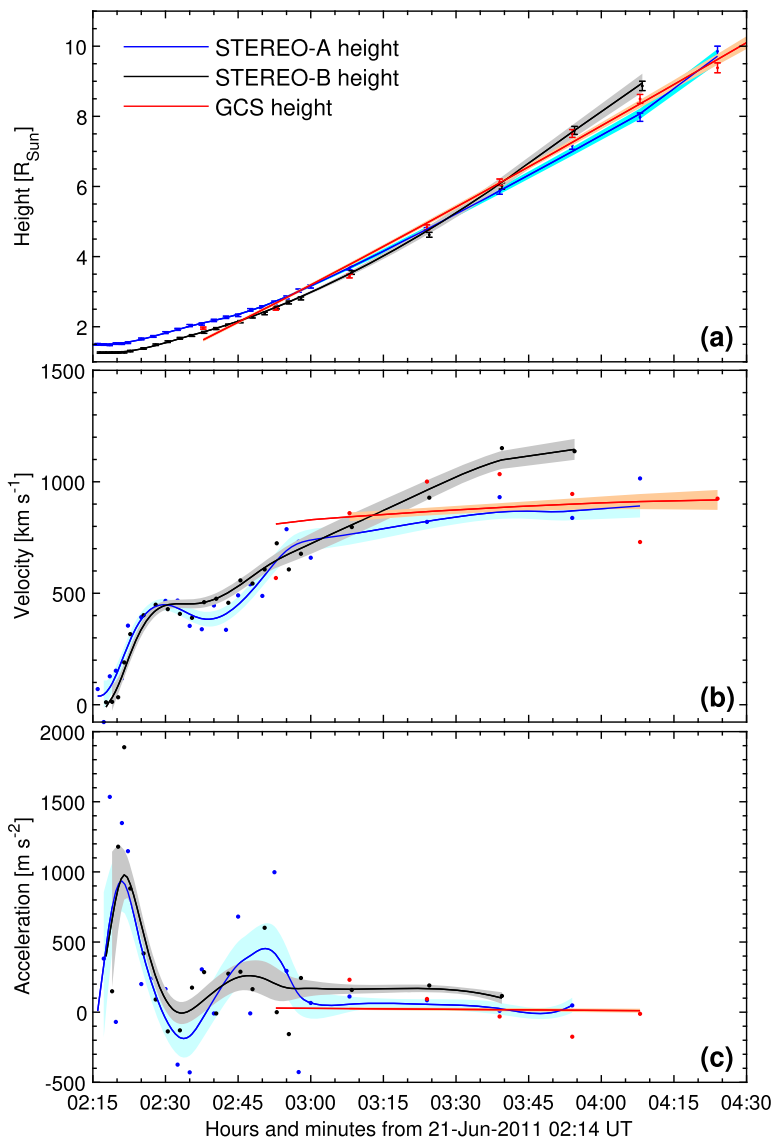

Figure 9 CME kinematics up to a height of $10 \mathrm{R}_{\odot}$. From top to bottom: height, velocity, acceleration as function of time. The dots are the measured points and direct time derivatives, the solid lines are the fits to the measurements and the time derivatives of these fits. The different colors represent the different sources from which the kinematics were obtained: STEREO-A (blue), STEREO-B (black) and GCS (red). The shadowed areas represent the uncertainties.

\section{Effects on the Magnetosphere}

Finally we analyze the geomagnetic response. In Figure 10, we show the sym-H storm index, the auroral electrojet $\mathrm{AE}$ and $\mathrm{AL}$ indices and the PCN-north index. The sym-H panel also shows the correction due to the magnetopause currents (blue trace). The sym-H index shows fairly quiet conditions. Interestingly, correcting for the contribution of the magnetopause currents (sym- $\mathrm{H}^{*}$ ) almost doubles the effects, leading to a moderate storm at the high speed stream before the shock. Basically this is the effect of the relatively high dynamic pressure (see Figure 1). Another moderate storm is caused by the CME sheath.

The AL and AE indices show signatures of a substorm at about $01 \mathrm{UT}$, June 23. At this time the ionospheric convection shows an increase (PCN index). This convection enhancement is likely being contributed from the nightside source of substorm activity (Sandholt, Andalsvik, and Farrugia, 2012). Some intermittent substorm activity is evident at the end of the interval. 


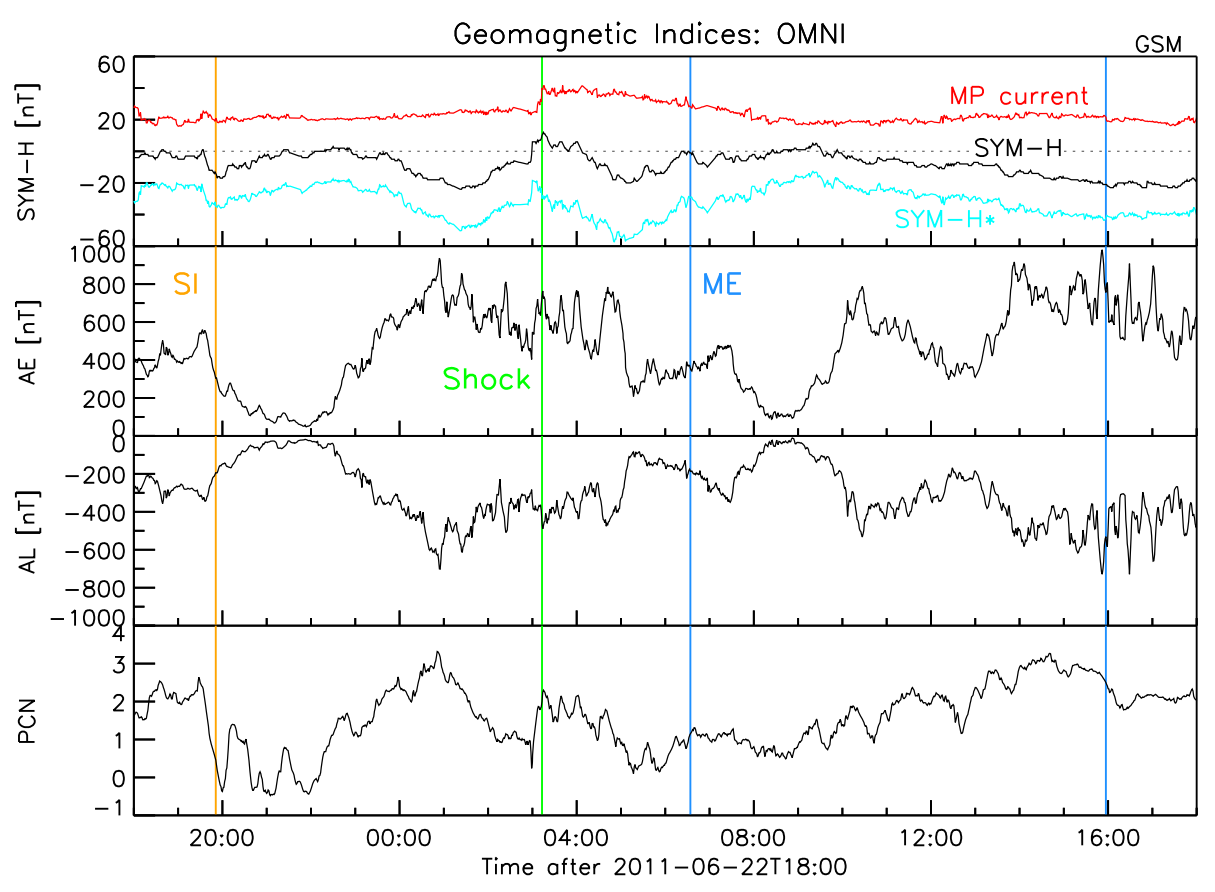

Figure 10 Geomagnetic indices for the same time interval and including the same vertical guidelines as shown in Figure 1. From top to bottom: SYM-H (black) including the magnetopause currents (red) and the resulting corrected SYM-H* (cyan), the Auroral Electrojet indices AE and AL and the Polar Cap Magnetic index $(\mathrm{PCN})$.

As Figure 1 shows, the dynamic pressure is higher than typical (i.e. $2 \mathrm{nPa}$ ). In addition, the $\mathrm{B}_{\mathrm{z}}$ component oscillates with large amplitudes (about $5 \mathrm{nT}$ ) in the sheath region of the CME. These two factors are bound to have an effect on the magnetopause (MP) shape when we consider the Shue et al. (1998) model which includes both the compression/rarefaction due to the dynamic pressure $\left(P_{\mathrm{dyn}}\right)$ and the erosion due to the negative $\mathrm{B}_{\mathrm{z}}$ component of the magnetic field. This result is confirmed in Figure 11, which shows from top to bottom the temporal profile of subsolar magnetopause position, the dawn-dusk terminator and their ratio, i.e. the aspect ratio of the magnetosphere. The subsolar distance (red) is closer to Earth than typical (i.e. $12 \mathrm{R}_{\mathrm{E}}$ ). A large Earthward shift occurs at shock arrival. Thereafter, the magnetosphere generally expands slowly. The aspect ratio is typically 1.5 . However, it changes significantly during the CME sheath passage visualizing the flaring of the magnetosphere. We conclude that the major effects were those produced by the sheath region.

\section{Discussion}

In this paper, we present a detailed case study of a CME ejected on June 21, 2011, using multi-viewpoint remote sensing and in situ observations supported by modeling to better understand and explain the intriguing in situ solar wind signatures observed as a consequence of the $\mathrm{CME}$ interacting with a nearby $\mathrm{CH}$. We find that the local and global magnetic field configuration, especially large-scale open magnetic structures such as $\mathrm{CHs}$, have a major effect on the early propagation direction of the CME. The low-lying, local magnetic field 


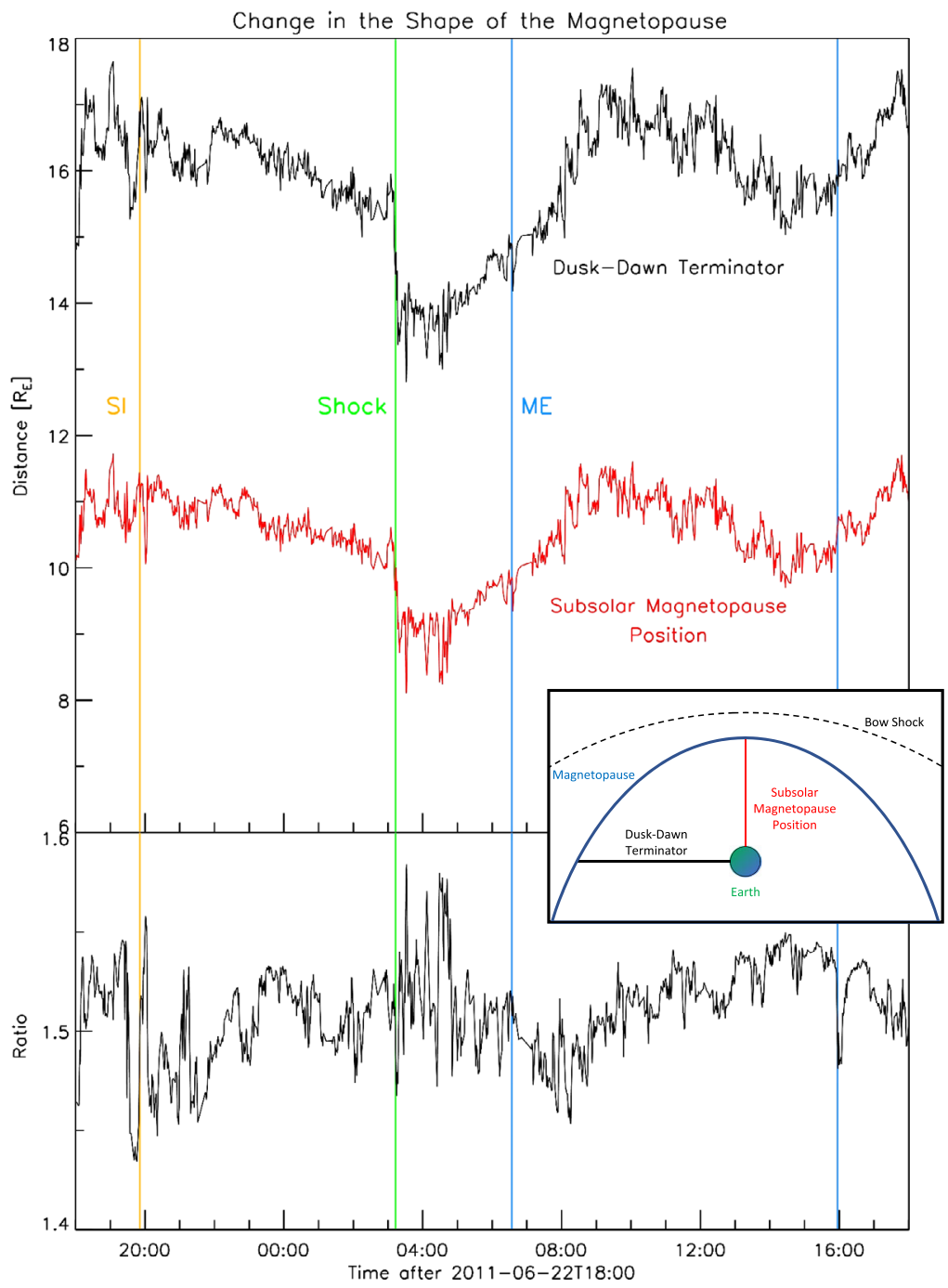

Figure 11 Temporal profile of the magnetopause shape for the same time interval and including the same vertical guidelines as shown in Figure 1. In the top panel the dusk-dawn terminator (black) and the subsolar magnetopause position (red) are shown. The bottom panel shows their ratio. The inset shows a cartoon demonstrating the shape of Earth's magnetosphere in regards to the dusk-dawn terminator and the subsolar magnetopause position.

configuration of the AR and its vicinity first leads to a non-radial ejection southwards. The global magnetic field structure, which in the close proximity is dominated by the open field lines of the $\mathrm{CH}$, causes the subsequent deflection of the CME north-eastwards.

From ForeCAT model results, we find that the open magnetic structures southwards and to the west of the AR seem to exert magnetic pressure on the CME, preventing it from propagating and expanding in these directions. The ForeCAT ensemble results show a larger spread in latitude than longitude, this is because the ensemble runs are based on how well the initial position within an active region can be identified. The spread in latitude and lon- 
gitude in the corona is simply a result of how sensitive the model is to the precise initial position, and in this case the magnetic forces consistently give a strong eastward deflection but the latitudinal motion is slightly more varied. The resulting propagation direction is also supported by the NLFFF model results. Cremades, Bothmer, and Tripathi (2006), Gopalswamy et al. (2009), Mäkelä et al. (2013) found that there is a correlation between a CME's direction and the properties of nearby $\mathrm{CHs}$ (distance, area and mean magnetic field strength). For the CME under study, we find that the direction in which the CME is ejected is mainly affected by the configuration of the local magnetic field at low heights and the position of the flux rope (as estimated from EUV observations) rather than the nearby $\mathrm{CH}$. The initial propagation of the CME to the south can be well observed off-limb in STB EUV images. The on-disk observations from the evolution of the coronal dimming associated with CME show the same behavior and can therefore be used as a proxy to derive the CME propagation direction (see also Dissauer et al., 2019). A similar conclusion is drawn by Mandrini et al. (2007), who analyzed the coronal dimming of the X17 flare event on October 28, 2003.

Starting at a height of $\sim 1.3 \mathrm{R}_{\odot}$ effects of the open field of the $\mathrm{CH}$ on the CME are clearly revealed. As the CME runs into the open field of the $\mathrm{CH}$, the CME's further expansion and propagation in the direction of the open field is prohibited, resulting in a deflection towards north and east. This can be interpreted such that the southward motion is actually stopped and reversed by the compressed magnetic field of the HSS. While in the ForeCAT model this occurs as a net northward motion of the entire structure, in reality, where the CME is not forced to maintain a uniform shape, it could manifest as an asymmetric expansion in the northward direction. Separating these effects requires comparison of the external deflection forces and the internal forces that maintain a coherent CME structure, which is beyond the scope of this work. Wang et al. (2011) defined three groups of deflected CMEs: asymmetrical expansion, non-radial ejection, and deflected propagation. Our event can be classified as a combination of two different processes. Firstly, the CMEs initial direction deviates from the radial direction and can be classified as a non-radial eruption (second group). And secondly, due to the presence of an ambient magnetic structure the CME is deflected and as such belongs to the third group (deflected propagation).

The in situ measured data near and at Earth show interesting signatures of the interaction between a SIR and a CME that occurred in the interplanetary space between the Sun and Earth. On June 22, 2011, at around 20:00 UT a clear stream interface signature can be identified, followed on June 23, 2011, at 03:15 UT by the HSS that also reveals a clear shock signature. The shock coincides with typical CME signatures starting at 06:30 UT, hence, can be attributed to be CME related. This, however, raises the question how that shock signature was produced. As shown in Figure 1, the speed of the CME matches the bulk speed of the HSS before the shock arrival which suggests that the shock is not driven by the CME at this point. But the short duration of the shock-sheath region of about $3 \mathrm{hrs}$ suggests otherwise. Due to the low compression of the magnetic field $(\sim 1.5)$ and of the density $(\sim 2)$ we can characterize the shock to have medium strength. We calculated the shock normal speed to be $\sim 700 \mathrm{~km} \mathrm{~s}^{-1}$ and found that the shock can be considered quasi-parallel.

Due to the inconclusive results in deriving the flux rope geometry using the Lundquist force-free flux rope model we are not able to reliably determine the part of the structure which the spacecraft intersected. However, we can estimate a trajectory from the modeled size and the propagation direction. As such, the most plausible interpretation is that the spacecraft only skims the outer edge of the CME and therefore the higher speed component, which drives the shock, is not observed in situ. This interpretation is consistent with the steadily decreasing magnetic strength profile (as opposed to peaking later). As shown before, due to the magnetic configuration and the interaction with the HSS, the CME does not 
follow a radial direction but deviates at least $30^{\circ}$ from it. This could explain the relative short standoff distance (e.g., shock-sheath duration) as the shock could still be driven at the apex but the measured bulk speed at the intersection trajectory is equal to the speed of the HSS. In other words, the shock at the spacecraft crossing is being driven but not by the part of the CME at this trajectory but rather by the plasma streaming from the apex.

Based on these results, we suspect that the CME is engulfed by the HSS during most of its propagation to $1 \mathrm{AU}$, which is consistent with the results from of the flux rope evolution. The flux tube expansion factor is with values of measured $n_{A}=0.51_{+0.14}^{-0.13}$ at the lower end of the range obtained by statistical studies (see DeVore, 2000; Gulisano et al., 2012; Qiu et al., 2007; Temmer et al., 2017). Possible interpretations are that the FRs expansion was hindered and/or that only part of the magnetic structure is encountered by the spacecraft. We suspect both to have happened in this case.

The effects of this interaction on the Earth's magnetosphere are weak-to-moderate but show some surprising features such as a strong compression of the magnetorsphere and significant flaring during the CME sheath passage. We find some normal storm as well as substorm activity caused by the CIR. Due to the unusual high dynamic pressure of the shock, for a weak CME, the subsolar position of the magnetopause is decreased up to $27 \%$ during the shock arrival, which causes significant magnetopause currents. This leads to the storm activity to be considered moderate.

\section{Summary and Conclusions}

We studied the origin of a peculiar in situ signature measured at 1 AU, caused by an Earthdirected ICME and its interaction with a nearby HSS with the aim to address two major questions: How such a unique in situ signature could be formed and whether the observed shock was still driven. Using a combination of observations and modeling efforts we were able to create a consistent interpretation of the event answering these questions:

- The CME is launched non-radially in south-eastern direction due to the configuration of the local magnetic field. This is reflected in the evolution of the dimming regions, the CME loops low in the corona (as seen in EUV observations) and the ForeCAT results.

- At a height after $1.3 \mathrm{R}_{\odot}$ the CME runs into the HSS which slows down the southward propagation until it is reversed and the CME is propagating northward. This is indicated by both the GCS measurements and the ForeCAT model. However, to be consistent with the GCS results, the ForeCAT model input requires an artificially scaled background field and a wider CME (overexpansion of CME cross section). With this the ForeCAT model mimics the effects of compression of the magnetic field due to the CME, which compresses the CH's open field and is then deflected away from it.

- Due to deflection and the HSS "wrapping" around the CME, we measure a relatively small FR size and possible slow expansion (owing to the estimated low-value $n_{A}=0.51_{+0.14}^{-0.13}$ ).

- The short standoff distance as well as the shock signature may be interpreted such as that the CME, which is engulfed by the HSS, only skims the spacecraft so its high speed part might have been missed. This interpretation is also consistent with the shape of the magnetic field profile which is steadily decreasing and shows no clear peak as expected from a central flux rope crossing. Due to the magnetic configuration and the interaction with the HSS, the CME does not follow a radial direction but deviates at least $30^{\circ}$ from it, causing this geometric effect in the measurements. 
Acknowledgements Open access funding provided by University of Graz. The SDO and STEREO image data and the Wind, ACE and STEREO in situ data is available by courtesy of NASA and the respective science teams. We also thank the OMNI website at Goddard Space Flight Center, USA for a significant portion of the data used. S.G.H., M.T., K.D. and A.M.V. acknowledge funding by the Austrian Space Applications Programme of the Austrian Research Promotion Agency FFG (ASAP-13 859729 SWAMI, ASAP-11 4900217 CORDIM and ASAP-14 865972 SSCME). S.J.H. acknowledges support from the JungforscherInnenfonds der Steiermärkischen Sparkassen. C.J.F. acknowledges support from NASA Wind NNX16AO04G and STEREO Quadrature grant. N.L. and C.J.F. acknowledge support from NSF grant AGS-1435785. F.C. acknowledges the support by FEDER/Ministerio de Ciencia, Innovación y Universidades - Agencia Estatal de Investigación/Proyecto (ESP2017-88436-R) and Predoctoral Research Grants 2016-MCIU/FSE (BES-2016077267). T.W. acknowledges DLR-grant 50 OC 1701 and DFG-grant WI3211/4-1. We thank the anonymous referee for constructive comments, which helped to improve the manuscript.

Disclosure of Potential Conflicts of Interest The authors declare that they have no conflicts of interest.

Publisher's Note Springer Nature remains neutral with regard to jurisdictional claims in published maps and institutional affiliations.

Open Access This article is distributed under the terms of the Creative Commons Attribution 4.0 International License (http://creativecommons.org/licenses/by/4.0/), which permits unrestricted use, distribution, and reproduction in any medium, provided you give appropriate credit to the original author(s) and the source, provide a link to the Creative Commons license, and indicate if changes were made.

\section{References}

Abraham-Shrauner, B.: 1972, Determination of magnetohydrodynamic shock normals. J. Geophys. Res. 77(4), 736. DOI. ADS.

Abraham-Shrauner, B., Yun, S.H.: 1976, Interplanetary shocks seen by AMES plasma probe on Pioneer 6 and 7. J. Geophys. Res. 81, 2097. DOI. ADS.

Acuña, M.H., Ogilvie, K.W., Baker, D.N., Curtis, S.A., Fairfield, D.H., Mish, W.H.: 1995, The global geospace science program and its investigations. Space Sci. Rev. 71, 5. DOI. ADS.

Alves, M.V., Echer, E., Gonzalez, W.D.: 2006, Geoeffectiveness of corotating interaction regions as measured by Dst index. J. Geophys. Res. 111, A07S05. DOI. ADS.

Brueckner, G.E., Howard, R.A., Koomen, M.J., Korendyke, C.M., Michels, D.J., Moses, J.D., Socker, D.G., Dere, K.P., Lamy, P.L., Llebaria, A., Bout, M.V., Schwenn, R., Simnett, G.M., Bedford, D.K., Eyles, C.J.: 1995, The Large Angle Spectroscopic Coronagraph (LASCO). Solar Phys. 162, 357. DOI. ADS.

Carcaboso Morales, F., Gómez-Herrero, R., Hidago, M.Á., Espinosa, F., Cernuda, I., Rodríguez-Pacheco, J.: 2018, Suprathermal electrons bidirectionality study inside magnetic clouds observed by STEREO (2007 - 2014). In: Solar Heliospheric and INterplanetary Environment (SHINE 2018), 81. ADS.

Colburn, D.S., Sonett, C.P.: 1966, Discontinuities in the solar wind. Space Sci. Rev. 5(4), 439. DOI. ADS.

Couvidat, S., Schou, J., Hoeksema, J.T., Bogart, R.S., Bush, R.I., Duvall, T.L., Liu, Y., Norton, A.A., Scherrer, P.H.: 2016, Observables processing for the helioseismic and magnetic imager instrument on the Solar Dynamics Observatory. Solar Phys. 291, 1887. DOI. ADS.

Cremades, H., Bothmer, V., Tripathi, D.: 2006, Properties of structured coronal mass ejections in solar cycle 23. Adv. Space Res. 38, 461. DOI. ADS.

Dasso, S., Mandrini, C.H., Démoulin, P., Luoni, M.L.: 2006, A new model-independent method to compute magnetic helicity in magnetic clouds. Astron. Astrophys. 455(1), 349. DOI. ADS.

Démoulin, P., Nakwacki, M.S., Dasso, S., Mandrini, C.H.: 2008, Expected in situ velocities from a hierarchical model for expanding interplanetary coronal mass ejections. Solar Phys. 250, 347. DOI. ADS.

DeVore, C.R.: 2000, Magnetic helicity generation by solar differential rotation. Astrophys. J. 539, 944. DOI. ADS.

Dissauer, K., Veronig, A.M., Temmer, M., Podladchikova, T., Vanninathan, K.: 2018, On the detection of coronal dimmings and the extraction of their characteristic properties. Astrophys. J. 855, 137. DOI. ADS.

Dissauer, K., Veronig, A.M., Temmer, M., Podladchikova, T.: 2019, Statistics of coronal dimmings associated with coronal mass ejections. II. Relationship between coronal dimmings and their associated CMEs. Astrophys. J. 874, 123. DOI. ADS.

Domingo, V., Fleck, B., Poland, A.I.: 1995, The SOHO mission: an overview. Solar Phys. 162, 1. DOI. ADS. 
Dumbović, M., Heber, B., Vršnak, B., Temmer, M., Kirin, A.: 2018, An analytical diffusion-expansion model for forbush decreases caused by flux ropes. Astrophys. J. 860, 71. DOI. ADS.

Farrugia, C.J., Burlaga, L.F., Lepping, R.P.: 1997, Magnetic Clouds and the Quiet-Storm Effect at Earth. Geophysical Monograph Series 98, American Geophysical Union, Washington, 91. DOI. ADS.

Gopalswamy, N., Mäkelä, P., Xie, H., Akiyama, S., Yashiro, S.: 2009, CME interactions with coronal holes and their interplanetary consequences. J. Geophys. Res. 114, A00A22. DOI. ADS.

Gosling, J.T., Baker, D.N., Bame, S.J., Feldman, W.C., Zwickl, R.D., Smith, E.J.: 1987, Bidirectional solar wind electron heat flux events. J. Geophys. Res. 92(A8), 8519. DOI.

Gulisano, A.M., Démoulin, P., Dasso, S., Rodriguez, L.: 2012, Expansion of magnetic clouds in the outer heliosphere. Astron. Astrophys. 543, A107. DOI. ADS.

He, W., Liu, Y.D., Hu, H., Wang, R., Zhao, X.: 2018, A stealth CME bracketed between slow and fast wind producing unexpected geoeffectiveness. Astrophys. J. 860, 78. DOI. ADS.

Howard, R.A., Moses, J.D., Vourlidas, A., Newmark, J.S., Socker, D.G., Plunkett, S.P., Korendyke, C.M., Cook, J.W., Hurley, A., Davila, J.M., Thompson, W.T., St Cyr, O.C., Mentzell, E., Mehalick, K., Lemen, J.R., Wuelser, J.P., Duncan, D.W., Tarbell, T.D., Wolfson, C.J., Moore, A., Harrison, R.A., Waltham, N.R., Lang, J., Davis, C.J., Eyles, C.J., Mapson-Menard, H., Simnett, G.M., Halain, J.P., Defise, J.M., Mazy, E., Rochus, P., Mercier, R., Ravet, M.F., Delmotte, F., Auchere, F., Delaboudiniere, J.P., Bothmer, V., Deutsch, W., Wang, D., Rich, N., Cooper, S., Stephens, V., Maahs, G., Baugh, R., McMullin, D., Carter, T.: 2008, Sun Earth connection coronal and heliospheric investigation (SECCHI). Space Sci. Rev. 136, 67. DOI. ADS.

Hudson, H.S., Lemen, J.R., Webb, D.F.: 1996, Coronal X-ray dimming in two limb flares. In: Bentley, R.D., Mariska, J.T. (eds.) Astronomical Society of the Pacific Conference Series, 111, 379. ADS.

Isavnin, A., Vourlidas, A., Kilpua, E.K.J.: 2013, Three-dimensional evolution of erupted flux ropes from the Sun $\left(2-20 \mathrm{R}_{\odot}\right)$ to 1 AU. Solar Phys. 284(1), 203. DOI. ADS.

Kaiser, M.L., Kucera, T.A., Davila, J.M., St. Cyr, O.C., Guhathakurta, M., Christian, E.: 2008, The STEREO mission: an introduction. Space Sci. Rev. 136, 5. DOI. ADS.

Kay, C., Gopalswamy, N.: 2018, The effects of uncertainty in initial CME input parameters on deflection, rotation, $\mathrm{B}_{z}$, and arrival time predictions. J. Geophys. Res. 123, 7220. DOI. ADS.

Kay, C., Opher, M., Evans, R.M.: 2013, Forecasting a coronal mass ejection's altered trajectory: ForeCAT. Astrophys. J. 775, 5. DOI. ADS.

Kay, C., Opher, M., Evans, R.M.: 2015, Global trends of CME deflections based on CME and solar parameters. Astrophys. J. 805, 168. DOI. ADS.

Kilpua, E., Koskinen, H.E.J., Pulkkinen, T.I.: 2017, Coronal mass ejections and their sheath regions in interplanetary space. Living Rev. Solar Phys. 14, 5. DOI. ADS.

Lavraud, B., Ruffenach, A., Rouillard, A.P., Kajdic, P., Manchester, W.B., Lugaz, N.: 2014, Geo-effectiveness and radial dependence of magnetic cloud erosion by magnetic reconnection. J. Geophys. Res. 119, 26. DOI. ADS.

Lemen, J.R., Title, A.M., Akin, D.J., Boerner, P.F., Chou, C., Drake, J.F., Duncan, D.W., Edwards, C.G., Friedlaender, F.M., Heyman, G.F., Hurlburt, N.E., Katz, N.L., Kushner, G.D., Levay, M., Lindgren, R.W., Mathur, D.P., McFeaters, E.L., Mitchell, S., Rehse, R.A., Schrijver, C.J., Springer, L.A., Stern, R.A., Tarbell, T.D., Wuelser, J.-P., Wolfson, C.J., Yanari, C., Bookbinder, J.A., Cheimets, P.N., Caldwell, D., Deluca, E.E., Gates, R., Golub, L., Park, S., Podgorski, W.A., Bush, R.I., Scherrer, P.H., Gummin, M.A., Smith, P., Auker, G., Jerram, P., Pool, P., Soufli, R., Windt, D.L., Beardsley, S., Clapp, M., Lang, J., Waltham, N.: 2012, The Atmospheric Imaging Assembly (AIA) on the Solar Dynamics Observatory (SDO). Solar Phys. 275, 17. DOI. ADS.

Liu, Y.D., Luhmann, J.G., Kajdič, P., Kilpua, E.K.J., Lugaz, N., Nitta, N.V., Möstl, C., Lavraud, B., Bale, S.D., Farrugia, C.J., Galvin, A.B.: 2014, Observations of an extreme storm in interplanetary space caused by successive coronal mass ejections. Nat. Commun. 5, 3481. DOI. ADS.

Lopez, R.E.: 1987, Solar cycle invariance in solar wind proton temperature relationships. J. Geophys. Res. 92, 11189. DOI. ADS.

Lugaz, N., Farrugia, C.J., Smith, C.W., Paulson, K.: 2015, Shocks inside CMEs: a survey of properties from 1997 to 2006. J. Geophys. Res. 120, 2409. DOI. ADS.

Mäkelä, P., Gopalswamy, N., Xie, H., Mohamed, A.A., Akiyama, S., Yashiro, S.: 2013, Coronal hole influence on the observed structure of interplanetary CMEs. Solar Phys. 284, 59. DOI. ADS.

Manchester, W.B. IV, Gombosi, T.I., De Zeeuw, D.L., Sokolov, I.V., Roussev, I.I., Powell, K.G., Kóta, J., Tóth, G., Zurbuchen, T.H.: 2005, Coronal mass ejection shock and sheath structures relevant to particle acceleration. Astrophys. J. 622, 1225. DOI. ADS.

Manchester, W.B., Gombosi, T.I., Roussev, I., Ridley, A., de Zeeuw, D.L., Sokolov, I.V., Powell, K.G., Tóth, G.: 2004, Modeling a space weather event from the Sun to the Earth: CME generation and interplanetary propagation. J. Geophys. Res. 109, A02107. DOI. ADS. 
Mandrini, C.H., Nakwacki, M.S., Attrill, G., van Driel-Gesztelyi, L., Démoulin, P., Dasso, S., Elliott, H.: 2007, Are CME-related dimmings always a simple signature of interplanetary magnetic cloud footpoints? Solar Phys. 244, 25. DOI. ADS.

Montgomery, M.D., Asbridge, J.R., Bame, S.J., Feldman, W.C.: 1974, Solar wind electron temperature depressions following some interplanetary shock waves: evidence for magnetic merging? J. Geophys. Res. 79(22), 3103. DOI.

Nieves-Chinchilla, T., Colaninno, R., Vourlidas, A., Szabo, A., Lepping, R.P., Boardsen, S.A., Anderson, B.J., Korth, H.: 2012, Remote and in situ observations of an unusual Earth-directed coronal mass ejection from multiple viewpoints. J. Geophys. Res. 117, A06106. DOI. ADS.

Paschmann, G., Daly, P.W.: 1998, Analysis Methods for Multi-Spacecraft Data. ISSI Scientific Report 1, ESA/ISSI, ISBN 1608-280X, ADS.

Pesnell, W.D., Thompson, B.J., Chamberlin, P.C.: 2012, The Solar Dynamics Observatory (SDO). Solar Phys. 275, 3. DOI. ADS.

Podladchikova, T., Van der Linden, R., Veronig, A.M.: 2017, Sunspot number second differences as a precursor of the following 11-year sunspot cycle. Astrophys. J. 850, 81. DOI. ADS.

Qiu, J., Hu, Q., Howard, T.A., Yurchyshyn, V.B.: 2007, On the magnetic flux budget in low-corona magnetic reconnection and interplanetary coronal mass ejections. Astrophys. J. 659, 758. DOI. ADS.

Richardson, I.G.: 2018, Solar wind stream interaction regions throughout the heliosphere. Living Rev. Solar Phys. 15, 1. DOI. ADS.

Richardson, I.G., Cane, H.V.: 2010, Near-Earth interplanetary coronal mass ejections during Solar Cycle 23 (1996-2009): catalog and summary of properties. Solar Phys. 264, 189. DOI. ADS.

Riley, P., Crooker, N.U.: 2004, Kinematic treatment of coronal mass ejection evolution in the solar wind. Astrophys. J. 600(2), 1035. DOI. ADS.

Ruffenach, A., Lavraud, B., Owens, M.J., Sauvaud, J.-A., Savani, N.P., Rouillard, A.P., Démoulin, P., Foullon, C., Opitz, A., Fedorov, A.: 2012, Multispacecraft observation of magnetic cloud erosion by magnetic reconnection during propagation. J. Geophys. Res. 117(A9), A09101. DOI. ADS.

Ruffenach, A., Lavraud, B., Farrugia, C.J., Démoulin, P., Dasso, S., Owens, M.J., Sauvaud, J.-A., Rouillard, A.P., Lynnyk, A., Foullon, C.: 2015, Statistical study of magnetic cloud erosion by magnetic reconnection. J. Geophys. Res. 120(1), 43. DOI. ADS.

Russell, C.T., Shinde, A.A., Jian, L.: 2005, A new parameter to define interplanetary coronal mass ejections. Adv. Space Res. 35, 2178. DOI. ADS.

Sandholt, P.E., Andalsvik, Y.L., Farrugia, C.J.: 2012, The pulsed nature of the nightside contribution to polar cap convection: repetitive substorm activity under steady interplanetary driving. Ann. Geophys. 30, 1539. DOI. ADS.

Schatten, K.H., Ness, N.F., Wilcox, J.M.: 1968, Influence of a solar active region on the interplanetary magnetic field. Solar Phys. 5, 240. DOI. ADS.

Schou, J., Scherrer, P.H., Bush, R.I., Wachter, R., Couvidat, S., Rabello-Soares, M.C., Bogart, R.S., Hoeksema, J.T., Liu, Y., Duvall, T.L., Akin, D.J., Allard, B.A., Miles, J.W., Rairden, R., Shine, R.A., Tarbell, T.D., Title, A.M., Wolfson, C.J., Elmore, D.F., Norton, A.A., Tomczyk, S.: 2012, Design and ground calibration of the Helioseismic and Magnetic Imager (HMI) instrument on the Solar Dynamics Observatory (SDO). Solar Phys. 275, 229. DOI. ADS.

Shue, J.-H., Song, P., Russell, C.T., Steinberg, J.T., Chao, J.K., Zastenker, G., Vaisberg, O.L., Kokubun, S., Singer, H.J., Detman, T.R., Kawano, H.: 1998, Magnetopause location under extreme solar wind conditions. J. Geophys. Res. 103, 17691. DOI. ADS.

Sterling, A.C., Hudson, H.S.: 1997, Yohkoh SXT observations of X-ray "dimming" associated with a halo coronal mass ejection. Astrophys. J. Lett. 491, L55. DOI. ADS.

Stone, E.C., Frandsen, A.M., Mewaldt, R.A., Christian, E.R., Margolies, D., Ormes, J.F., Snow, F.: 1998, The advanced composition explorer. Space Sci. Rev. 86, 1. DOI. ADS.

Tadesse, T., Wiegelmann, T., Gosain, S., MacNeice, P., Pevtsov, A.A.: 2014, First use of synoptic vector magnetograms for global nonlinear, force-free coronal magnetic field models. Astron. Astrophys. 562, A105. DOI. ADS.

Temmer, M., Reiss, M.A., Nikolic, L., Hofmeister, S.J., Veronig, A.M.: 2017, Preconditioning of interplanetary space due to transient CME disturbances. Astrophys. J. 835, 141. DOI. ADS.

Thernisien, A.: 2011, Implementation of the graduated cylindrical shell model for the three-dimensional reconstruction of coronal mass ejections. Astrophys. J. Suppl. 194, 33. DOI. ADS.

Thernisien, A., Vourlidas, A., Howard, R.A.: 2009, Forward modeling of coronal mass ejections using STEREO/SECCHI data. Solar Phys. 256, 111. DOI. ADS.

Thernisien, A.F.R., Howard, R.A., Vourlidas, A.: 2006, Modeling of flux rope coronal mass ejections. Astrophys. J. 652, 763. DOI. ADS. 
Thompson, B.J., Plunkett, S.P., Gurman, J.B., Newmark, J.S., St. Cyr, O.C., Michels, D.J.: 1998, SOHO/EIT observations of an Earth-directed coronal mass ejection on May 12, 1997. Geophys. Res. Lett. 25, 2465. DOI. ADS.

Thompson, B.J., Cliver, E.W., Nitta, N., Delannée, C., Delaboudinière, J.-P.: 2000, Coronal dimmings and energetic CMEs in April-May 1998. Geophys. Res. Lett. 27, 1431. DOI. ADS.

Verbanac, G., Vršnak, B., Živković, S., Hojsak, T., Veronig, A.M., Temmer, M.: 2011, Solar wind high-speed streams and related geomagnetic activity in the declining phase of solar cycle 23. Astron. Astrophys. 533, A49. DOI. ADS.

Vršnak, B., Dumbović, M., Čalogović, J., Verbanac, G., Poljančić Beljan, I.: 2017, Geomagnetic effects of corotating interaction regions. Solar Phys. 292, 140. DOI. ADS.

Wang, Y., Shen, C., Wang, S., Ye, P.: 2004, Deflection of coronal mass ejection in the interplanetary medium. Solar Phys. 222(2), 329. DOI. ADS.

Wang, Y., Xue, X., Shen, C., Ye, P., Wang, S., Zhang, J.: 2006, Impact of major coronal mass ejections on geospace during 2005 September 7-13. Astrophys. J. 646(1), 625. DOI. ADS.

Wang, Y., Chen, C., Gui, B., Shen, C., Ye, P., Wang, S.: 2011, Statistical study of coronal mass ejection source locations: understanding CMEs viewed in coronagraphs. J. Geophys. Res. 116, A04104. DOI. ADS.

Wang, Y., Wang, B., Shen, C., Shen, F., Lugaz, N.: 2014a, Deflected propagation of a coronal mass ejection from the corona to interplanetary space. J. Geophys. Res. 119(7), 5117. DOI. ADS.

Wang, Y., Wang, B., Shen, C., Shen, F., Lugaz, N.: 2014b, Deflected propagation of a coronal mass ejection from the corona to interplanetary space. J. Geophys. Res. 119, 5117. DOI. ADS.

Wang, Y., Zhou, Z., Shen, C., Liu, R., Wang, S.: 2015, Investigating plasma motion of magnetic clouds at 1 AU through a velocity-modified cylindrical force-free flux rope model. J. Geophys. Res. 120(3), 1543. DOI. ADS.

Wang, Y., Zhang, Q., Liu, J., Shen, C., Shen, F., Yang, Z., Zic, T., Vrsnak, B., Webb, D.F., Liu, R.: 2016, On the propagation of a geoeffective coronal mass ejection during 15-17 March 2015. J. Geophys. Res. 121(8), 7423. DOI. ADS.

Wang, Y., Shen, C., Liu, R., Liu, J., Guo, J., Li, X., Xu, M., Hu, Q., Zhang, T.: 2018, Understanding the twist distribution inside magnetic flux ropes by anatomizing an interplanetary magnetic cloud. J. Geophys. Res. 123(5), 3238. DOI. ADS.

Wheatland, M.S., Sturrock, P.A., Roumeliotis, G.: 2000, An optimization approach to reconstructing forcefree fields. Astrophys. J. 540, 1150. DOI. ADS.

Wiegelmann, T.: 2007, Computing nonlinear force-free coronal magnetic fields in spherical geometry. Solar Phys. 240, 227. DOI. ADS.

Winslow, R.M., Lugaz, N., Schwadron, N.A., Farrugia, C.J., Yu, W., Raines, J.M., Mays, M.L., Galvin, A.B., Zurbuchen, T.H.: 2016, Longitudinal conjunction between MESSENGER and STEREO A: development of ICME complexity through stream interactions. J. Geophys. Res. 121, 6092. DOI. ADS.

Yermolaev, Y.I., Lodkina, I.G., Nikolaeva, N.S., Yermolaev, M.Y., Riazantseva, M.O., Rakhmanova, L.S.: 2018, Statistic study of the geoeffectiveness of compression regions CIRs and Sheaths. J. Atmos. SolarTerr. Phys. 180, 52. DOI. ADS.

Yurchyshyn, V.: 2008, Relationship between EIT posteruption arcades, coronal mass ejections, the coronal neutral line, and magnetic clouds. Astrophys. J. 675(1), L49. DOI. ADS.

Zhuang, B., Wang, Y., Hu, Y., Shen, C., Liu, R., Gou, T., Zhang, Q., Li, X.: 2019, Numerical simulations on the deflection of coronal mass ejections in the interplanetary space. Astrophys. J. 876(1), 73. DOI. ADS. 